\title{
RELATION BETWEEN THE COLLECTOR CURRENT AND THE TWO-DIMENSIONAL ELECTRON GAS STORED IN THE BASE-COLLECTOR HETEROJUNCTION NOTCH OF InAlAs/InGaAs/InAlGaAs DHBTs
}

\author{
CHAO-HSING HUANG, TSUEN-LIN LEE and HAO-HSIUNG LIN† \\ Room 419, Department of Electrical Engineering, National Taiwan University, \\ Taipei, Taiwan, R.O. China
}

(Received 18 November 1994; in revised form 20 December 1994)

\begin{abstract}
From the Gummel plots of InAlAs/InGaAs/ $/ \mathrm{In}_{0.52} \mathrm{Al}_{x} \mathrm{Ga}_{0.48}$. As double heterojunction bipolar transistors (DHBTs), the relationship between the collector current and the density of the two-dimensional electron gas (2DEG) stored in the base-collector $(B-C)$ heterojunction notch is found to be an exponential function which stems from the thermionic emission escaping process of the 2DEG.
\end{abstract}

\section{INTRODUCTION}

Two-dimensional electron gas (2DEG) stored in a heterojunction notch is always an interesting and important subject. The applications of this phenomenon have included various important semiconductor devices. One of the most important examples is high electron mobility transistor (HEMT), which has entered into our daily life. In heterojunction bipolar transistor (HBT), an emerging counterpart of HEMT, 2DEG also plays an important role on some of their characteristics. HBT with $2 \mathrm{DEG}$ emitter has been demonstrated[1,2]. In this design, 2DEG charges stored in emitter-base junction notch was used to attain low offset voltage, high current gain and offer high frequency performance. In InAlAs/InGaAs DHBT, it was found that an extra 2DEG stored in the $B-C$ heterojunction notch may push up the potential spike (2DEG effect) and cause a soft-rising output characteristics, i.e. the reachthrough effect[3].

In these HBT examples, the density of 2DEG is a crucial factor which is determined by the heterojunction band offset, the magnitude and capture rate of the current passing through it, and the doping concentration of the heterojunction. To understand the relationship between them is not only a step to design HBTs with abrupt heterojunction but also an interesting topic of carrier transport dynamics.

In this study, the relationship between the collector current and the density of the $2 \mathrm{DEG}$ stored in the base-collector $(B-C)$ heterojunction notch was extracted from the Gummel plots of InAlAs/InGaAs $/ \operatorname{In}_{0.52} \mathrm{Al}_{x} \mathrm{Ga}_{0.48-x}$ As DHBTs under

†To whom all correspondence should be addressed. several $B-C$ bias voltages. The result is an exponential function. By considering the balance between the trapping and escaping process of the $2 D E G$, we found the thermionic emission escaping process is the reason of the exponential behavior. HBTs with different InAlGaAs collector, i.e. with different band offset, were also studied. Conduction band offset is found to be a main factor to control the escaping barrier.

\section{EXPERIMENT}

The epilayers of the devices were grown on (100) InP substrate by using solid source molecular beam epitaxy (MBE). The dopant for $n$-type and $p$-type layers are $\mathrm{Si}$ and $\mathrm{Be}$, respectively. The layer structures are summarized in Table 1 . Notice that, between the InAlGaAs collector and the $p^{+}-$InGaAs base, there is a 300- $\AA$-thick undoped InGaAs spacer which is used to remove the reach-through effect. There are six devices being studied. They are R504, R505, R506, R507, R508 and R640. For devices R504-R508, the only difference between them is on the composition of their $\mathrm{In}_{0.52} \mathrm{Al}_{x} \mathrm{Ga}_{0.48-x} \mathrm{As}$ collector. Besides, $\mathrm{R} 640$ has an InAlAs collector and a different base doping concentration. The $\operatorname{In}_{0.52} \mathrm{Al}_{x} \mathrm{Ga}_{0.48-x}$ As collector composition, designed base doping concentration, and measured collector doping concentration are summarized in Table 2. The estimated collector band gap and base-collector conduction band offset of each device is also attached. The collector doping concentrations were measured from $C-V$ method.

To fabricate the DHBTs, an Au-Ge-Ni alloy was firstly deposited and lifted-off on the cap layer for the emitter ohmic contact. Solution $1 \mathrm{H}_{3} \mathrm{PO}_{4}: 1 \mathrm{H}_{2} \mathrm{O}_{2}: 20$ $\mathrm{H}_{2} \mathrm{O}$ was then used to remove both the cap and the 
Table 1. The layer structures of InAlAs/InGaAs $/ \operatorname{In}_{0.52} \mathrm{Al}_{x} \mathrm{Ga}_{0.48-x}$ As DHBTs

\begin{tabular}{|c|c|c|c|}
\hline Layer & Material & Thickness $(\mu \mathrm{m})$ & Doping $\left(\mathrm{cm}^{-3}\right)$ \\
\hline Cap 1 & InAs & 0.015 & $3 \times 10^{19}$ \\
\hline Cap 2 & InGaAs & 0.1 & $3 \times 10^{19}$ \\
\hline Cap 3 & InAlAs & 0.05 & $3 \times 10^{19}$ \\
\hline Emitter & InAlAs & 0.15 & $1 \times 10^{17}$ \\
\hline Spacer & InGaAs & 0.03 & undoped \\
\hline Base & InGaAs & 0.15 & - \\
\hline Spacer & InGaAs & 0.03 & undoped \\
\hline Collector & $\mathrm{In}_{0.52} \mathbf{A l}_{x} \mathbf{G a}_{0.48-x} \mathrm{As}$ & 0.4 & $\sim 7 \times 10^{16}$ \\
\hline Buffer & InGaAs & 0.2 & $3 \times 10^{19}$ \\
\hline Substrate & InP & 350 & $1 \times 10^{18}(n)$ \\
\hline
\end{tabular}

Table 2. The parameters of base and $\mathrm{In}_{0.52} \mathrm{Al}_{x} \mathrm{Ga}_{0.48-x}$ As collector

\begin{tabular}{lccccc}
\hline & & & & \multicolumn{2}{c}{ Doping $\left(\mathrm{cm}^{-3}\right)$} \\
\cline { 5 - 6 } Run No. & $\begin{array}{c}\text { Material } \\
(x)\end{array}$ & $E_{8}(\mathrm{eV})$ & $\Delta E_{\mathrm{C}}(\mathrm{eV})$ & Base & Collector \\
\hline R508 & 0.00 & 0.75 & 0.00 & $5 \times 10^{18}$ & $5.9 \times 10^{16}$ \\
R507 & 0.12 & 0.93 & 0.14 & $5 \times 10^{18}$ & $6.6 \times 10^{16}$ \\
R506 & 0.24 & 1.10 & 0.27 & $5 \times 10^{18}$ & $6.8 \times 10^{16}$ \\
R505 & 0.36 & 1.28 & 0.40 & $5 \times 10^{18}$ & $6.6 \times 10^{16}$ \\
R504 & 0.48 & 1.45 & 0.53 & $5 \times 10^{18}$ & $6.3 \times 10^{16}$ \\
R640 & 0.48 & 1.45 & 0.53 & $1.3 \times 10^{19}$ & $5.8 \times 10^{16}$ \\
\hline
\end{tabular}

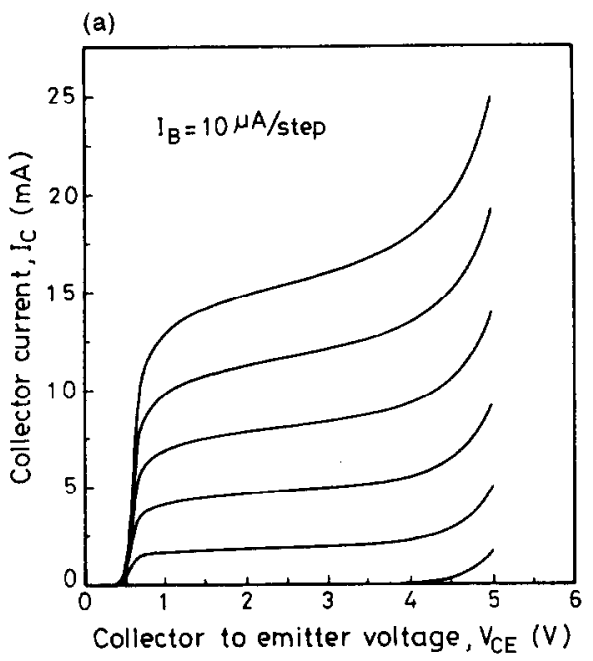

(b)

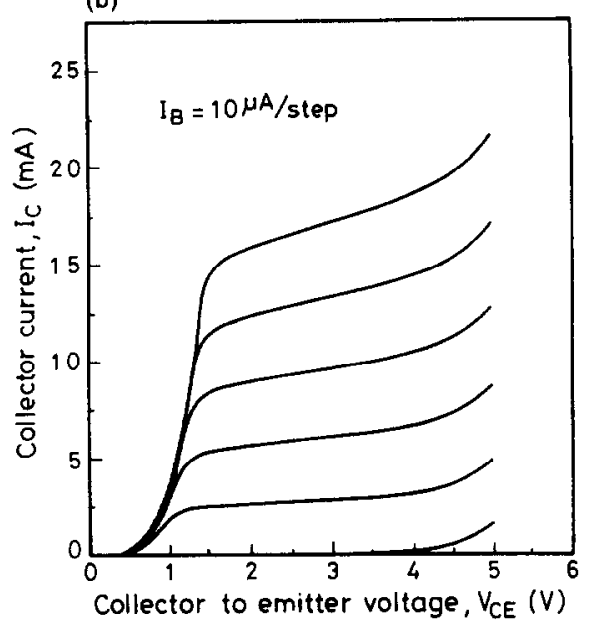

(c)

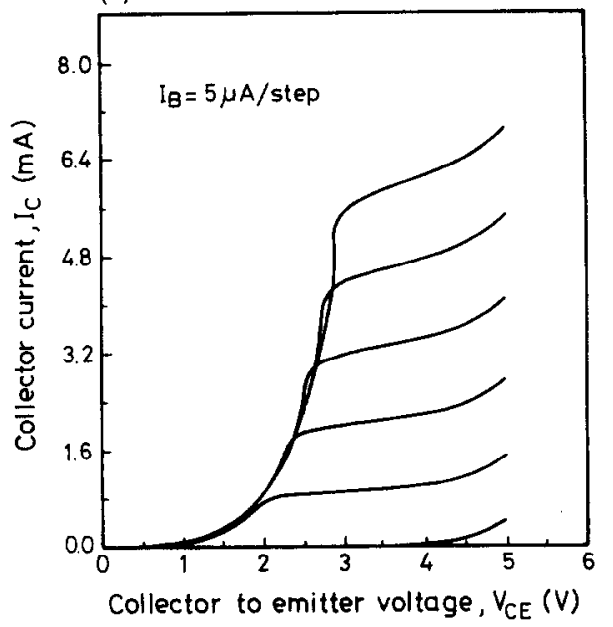

Fig. 1. The common emitter output characteristics of DHBT: (a) R506; (b) R505; and (c) R504, respectively. The $B-C$ conduction band offset of DHBT R506, R505 and R504 are $0.27,0.4$ and $0.53 \mathrm{eV}$, respectively. 
emitter layer to expose the base layer. The followed $\mathrm{Au} / \mathrm{Ti}$ nonalloyed contact was then deposited and lifted-off on the base layer. Finally, the devices were isolated by mesa etching in a $1 \mathrm{H}_{3} \mathrm{PO}_{4}: 1 \mathrm{H}_{2} \mathrm{O}_{2}: 20$ $\mathrm{H}_{2} \mathrm{O}$ solution. The emitter area of the device is $60 \times 60 \mu \mathrm{m}^{2}$.

\section{RESULTS AND DISCUSSIONS}

Figure 1(a), (b) and (c) show the common emitter output characteristics of DHBT R506, R505 and R504, respectively. As summarized in Table 2, their $B-C$ conduction band offset are $0.27,0.4$ and $0.53 \mathrm{eV}$, respectively. In Fig. 1(a), the large $0.37 \mathrm{~V}$ offset voltage is due to the existence of $E-B$ potential spike[4] which is preserved for hot electron launching. The sharp turn on collector current as can be seen in the saturation region indicates no reach-through effect in this device. In Fig. 1(b), the collector current of the device, R505, shows a soft turn on in its saturation region. Reach-through effect can be clearly identified. In Fig. 1(c), the device, R504, shows similar characteristics as R505. From the above results, it provides an important evidence for the band offset dependent $2 D E G$ effect, which will be discussed later.

The Gummel plots of R505 with several different $B-C$ junction reverse bias voltages $\left(V_{\mathrm{BC}}\right)$ are shown in Fig. 2. As can be seen, in low current region, $V_{\mathrm{BC}}$ has no effect on the collector current. But, in high current region, the collector current splits into several branches. The more negative the reverse bias voltage, $V_{\mathrm{BC}}$, the higher the collector current. This finding provides an important evidence for the currentdependent $2 \mathrm{DEG}$ effect. According to our calculation based on depletion approximation in which the 2DEG charge is not considered, when $V_{\mathrm{BC}}$ is smaller than $-0.2 \mathrm{~V}, 300$ - $\AA$-thick InGaAs narrow band gap spacer is thick enough to pull down the heterojunction spike and eliminate the reach through effect.

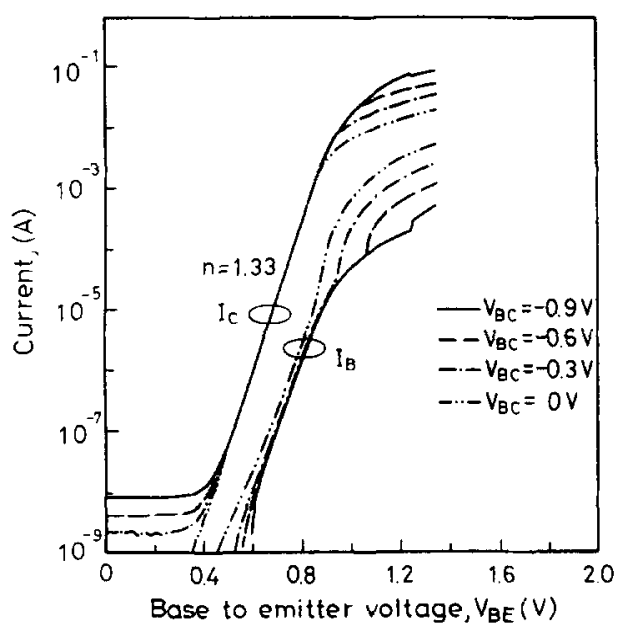

Fig. 2. The Gummel plot of DHBT R505 under different $B-C$ junction reverse bias voltages $\left(V_{\mathrm{BC}}\right)$. The $B-C$ conduction band offset of DHBT R505 is $0.4 \mathrm{eV}$.

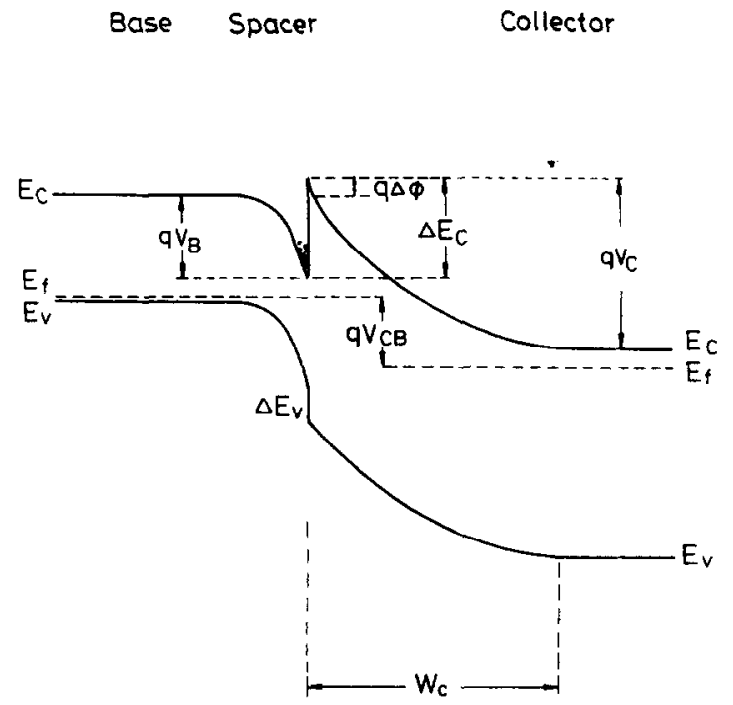

Fig. 3. The $B-C$ junction band diagram of DIIBT under the turning point condition.

Therefore, in the low current region with $V_{\mathrm{BC}}$ smaller than $-0.2 \mathrm{~V}$, the collector current and the base current are independent of the reverse $B-C$ bias voltage. When $V_{\text {BC }}$ is zero volts, our calculation indicates that the tip of the spike is $65 \mathrm{meV}$ higher than the conduction band minimum of the neutral base. This high spike begins to reflect injection carriers back to the base region and enhances the base recombination current as can be seen in Fig. 2. In this low current region, because the increase of $2 D E G$ charge in $E-B$ heterojunction due to the carrier reflection is still not comparable with the ion charges of the $E-B$ depletion region, the spike energy of $E-B$ heterojunction does not change. Since the collector current is controlled by the highest energy barrier it sees, i.e. the spike energy of $E-B$ heterojunction[5,6], the collector current is not different from those with $V_{\mathrm{BC}}$ smaller than $-0.2 \mathrm{~V}$. However, in high current region, care must be taken on the 2DEG charges, because their densities are comparable with the impurity ion charges. The collector current passing through the $B-C$ heterojunction notch may provide enough 2DEG charge to push up the spike and cause the 2DEG effect. When the spike is high enough to reflect the injection carriers, the minority carrier concentration in the base as well as the 2DEG density in the $E-B$ heterojunction notch increase. The former causes a sharp rising on the corresponding base current as shown in Fig. 2. The latter will push up the $E-B$ heterojunction spike, which will result in the decrease of the collector current. Furthermore, for the case of larger reverse $B-C$ bias voltage, it needs more $2 D E G$ density to push up the $B-C$ heterojunction spike to a certain level. Therefore, the decrease of the corresponding collector current takes place in higher current level.

Tiwari has presented similar Gummel plots[7], which were obtained from AlGaAs/GaAs DHBT 


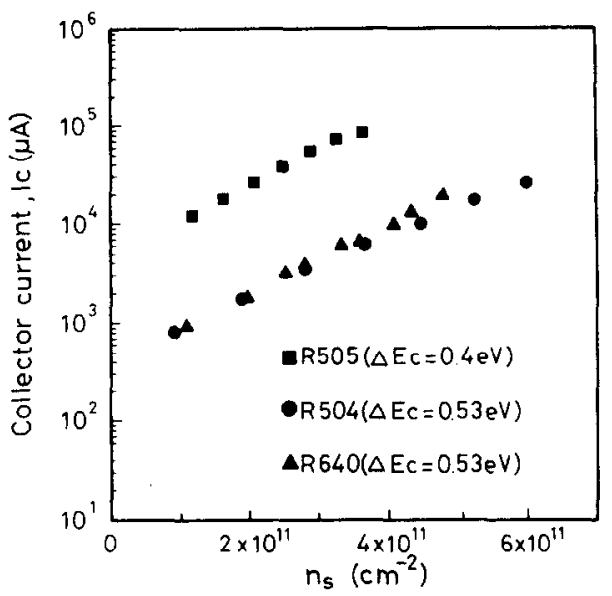

Fig. 4. The collector currents, $I_{C} s$ as functions of $2 D E G$ densities, $n_{\mathrm{s}} \mathrm{s}$. Circles, rectangles and triangles represent the data of DHBT R504, R505 and R640, respectively.

with graded base-collector junction. In his finding, the mobile charge from high collector current may push up the heterojunction spike and cause the soft-rising output characteristics. In our device, the 2DEG effect appears at a current density as low as $25-280 \mathrm{~A} / \mathrm{cm}^{2}$. At such low current density, the mobile charge is much lower than the impurity charges and the 2DEG charge in the depletion region. Therefore, the mobile charge effect and Kirk effect[8] are not considered in this study.

As the Gummel plots shown in Fig. 2, each current can be clearly divided into two parts on which the $B-C$ heterojunction spike does and does not cause the reach-through effect, respectively. The turning point of each current corresponds to a similar band structure as shown in Fig. 3. In this diagram, thermionic field emission (TFE) model[9] is considered, and the spike tip is higher than the conduction band minimum of the base neutral region. The difference, $\Delta \phi$, is just the effective barrier height lowering of the model. Although the spike tip is higher than the conduction band minimum of the base neutral region, the high tunneling rate due to the strong field around the spike tip prevents the reflection of the injection carriers. This effective barrier height lowering is relative to the electric field of the InAlGaAs side of the $B-C$ heterojunction and can be calculated from TFE model. The effect of $V_{\mathrm{BC}}$ on the band structure is mainly on the depletion width of the InAlGaAs side and the effective barrier height lowering. The latter is just slightly, therefore, the voltage drops across the InGaAs base and spacer for different $V_{\mathrm{BC}}$ are nearly the same. Since the band structures on the turning points can be calculated, the collector currents and bias voltages $V_{\mathrm{BC}} \mathrm{s}$ of these turning points provide us an opportunity to study the relationship between the current passing through a heterojunction notch and the 2DEG density stored in it.

The procedure used to calculate the 2DEG sheet charges density, $n_{\mathrm{s}}$, is described briefly as follows. In order to simplify the calculation, the 2DEG is assumed to be a delta function located on the heterojunction interface, and depletion approximation is used to find the electric field in the depletion region. The first step of the procedure is to select a possible voltage drop across the depletion region of InAIGaAs side, $V_{\mathrm{C}}$. Once the voltage drop is determined, the maximum electric field of the depletion region, $\epsilon_{\max }$, can be found by using depletion approximation. In turn, the effective barrier height lowering, $\Delta \phi$, can be found by using TFE model. And the voltage drop across the depletion region of the InGaAs side, $V_{B}$, is:

$$
V_{\mathrm{B}}=\frac{\Delta E_{\mathrm{C}}}{q}-\Delta \phi,
$$

where $\Delta E_{C}$ is the conduction band offset, and $q$ is unit charge. And the reverse bias voltage, $V_{\mathrm{BC}}$, is:

$$
V_{\mathrm{BC}}=-\left(V_{\mathrm{B}}+V_{\mathrm{C}}-V_{\mathrm{bi}}\right)
$$

where $V_{\mathrm{bi}}$ is the built-in potential of the junction. In the calculation, the possible $V_{\mathrm{C}}$ is readjusted to meet the required $V_{\mathrm{BC}}$. Once $V_{\mathrm{B}}$ and $V_{\mathrm{C}}$ are determined, charge densities of InGaAs side and InAlGaAs side, i.e. $Q_{\mathrm{B}}$ and $Q_{\mathrm{C}}$, can be easily found, and the 2DEG sheet density can be estimated from the equation:

$$
n_{\mathrm{s}}=\frac{Q_{\mathrm{c}}-Q_{\mathrm{B}}}{q} .
$$

Figure 4 shows the collector current versus 2DEG density plots of devices R504, R505 and R640. The most striking result from this figure is that the relationships between the collector current and the 2DEG density of these devices follow exponential law. In addition, the plots suggest that the smaller the $\Delta E_{\mathrm{C}}$, the larger the collector current for a given $n_{\mathrm{s}}$. These findings can be explained as follows. Figure 5 shows the schematic band diagram of the $B-C$ heterojunction notch. As shown in the figure, the supplying source of the 2DEG is the trapping of the collector current. And the trapping current, $I_{\mathrm{T}}$, is:

$$
I_{\mathrm{T}}=\gamma I_{\mathrm{C}},
$$

where $\gamma$ is the trapping efficiency. The loss of the 2DEG includes the recombination and thermionic
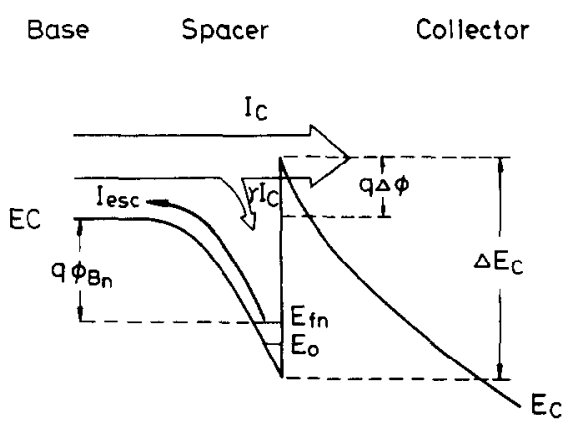

Fig. 5. The schematic band diagram shows the supplying current, escaping current, and emission barrier height of the 2DEG stored in a InAlGaAs/InGaAs heterojunction notch. 
emission of the 2DEG. The former can be neglected because of its magnitude, and the emission current, i.e. escaping current, $I_{\text {esc }}$, should be proportional to the barrier height, $\phi_{\mathrm{Bn}}$, seen by the 2DEG. As shown in the figure, the barrier height is:

$q \phi_{\mathrm{Bn}}=E_{\mathrm{C}}-E_{\mathrm{fn}}=\Delta E_{\mathrm{C}}-q \Delta \phi-\left(E_{\mathrm{fn}}-E_{0}\right)-E_{0}$,

where $E_{\mathrm{C}}$ is the conduction band minimum of the neutral base region, $E_{\ell_{\mathrm{n}}}$ is the quasi Fermi level of the 2DEG, and $E_{0}$ is the ground state of the heterojunction notch. And the escaping current of the $2 \mathrm{DEG}$ is:

$$
I_{\text {esc }} \propto \exp \left(-q \phi_{\mathrm{Bn}} / k T\right),
$$

where $k$ is Boltzmann's constant and $T$ is the absolute temperature. In steady state, the supplying rate should be equal to the emission rate, i.e. $I_{\mathrm{T}}=I_{\mathrm{esc}}$. And the collector current can be expressed as:

$$
I_{\mathrm{C}} \propto \frac{\exp \left(-q \phi_{\mathrm{Ba}} / k T\right)}{\gamma} .
$$

If $\gamma$ is nearly a constant, then $I_{C}$ is an exponential function of the emission barrier height, $\phi_{\mathrm{Bn}}$.

According to eqn (5), $\phi_{\mathrm{Bn}}$ has four items. The first item, $\Delta E_{C}$, is determined by the material compositions. The second item, $\Delta \phi$, can be found by using TFE model. The third item, $\left(E_{\mathrm{fn}}-E_{0}\right)$, is relative to the 2DEG density, $n_{\mathrm{s}}$, and can be solved from the following equation:

$$
n_{\mathrm{s}}=\int_{E_{0}}^{\infty} \frac{m^{*}}{\pi \hbar^{2}} \frac{\mathrm{d} E}{1+\exp \left(\frac{E-E_{\mathrm{fn}}}{k T}\right)},
$$

where $\hbar$ is the reduced Planck's contant. The final item, $E_{0}$, is a function of $\epsilon_{\max }$, and can be found from the well known solutions of Airy functions for triangular quantum well[10];

$$
E_{0}=\left(\frac{\hbar^{2} q^{2} \epsilon_{\max }^{2}}{2 m^{*}}\right)^{1 / 3}\left(\frac{9 \pi}{8}\right)^{2 / 3}
$$

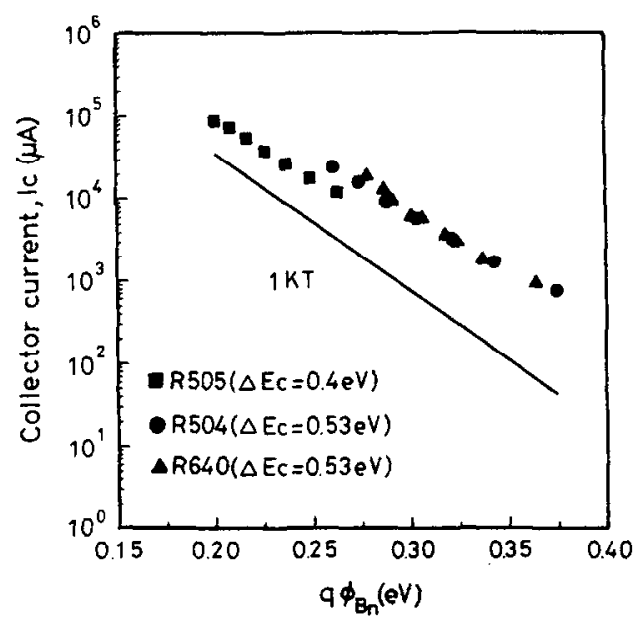

Fig. 6. The collector current $I_{C} s$ as functions of the emission barrier height $q \phi_{\mathrm{Bn}} \mathrm{s}$. Circles, rectangles and triangles represent the data of DHBT R504, R505 and R640, respectively. where $m^{*}$ is the electron effective mass. Since $\epsilon_{\max }$ and $n_{\mathrm{s}}$ are known, the emission barrier height, $\phi_{\mathrm{Bn}}$, can be calculated. In our calculation, for the sake of simplification, only the ground state of the quantum well is considered.

Figure 6 shows the collector current, $I_{\mathrm{C}}$, as a function of emission barrier height, $\phi_{\mathrm{Bn}}$. Data from devices with different $\Delta E_{\mathrm{C}}$ are plotted in the figure. Again, the relation is an exponential function. However, the most important is that the slope of the data is nearly $1 k T$. These results prove that the $2 \mathrm{DEG}$ supply current $I_{\mathrm{C}}$ is controlled by the thermionic emission process.

Based on this finding, it is very clearly that $\Delta E_{C}$ plays a very important role in the $2 D E G$ effect. The 2DEG in devices with smaller $\Delta E_{\mathrm{C}}$ has smaller emission barrier height, and needs higher supply current $I_{\mathrm{C}}$ to sustain a certain 2DEG density. This explains why our devices with $\Delta E_{\mathrm{C}}$ lower than $0.4 \mathrm{eV}$ do not have the reach-through effect. The reason is that their $\phi_{\mathrm{Bn}} \mathrm{s}$ are too small to prevent the escaping of 2DEG in normal operation current range. However, when the devices are driven to very high $I_{\mathrm{C}}$, the effect may reappear.

There is one interesting point in Fig. 6. As can be seen, for the same $\phi_{\mathrm{Bn}}$, the collector current of device R505 is smaller than those of device R504 and R640. A possible reason is that the electric field applied on the depletion region of the InGaAs side under the turning point condition is relative to the conduction band offset. For device R505, its band offset is $0.4 \mathrm{eV}$, and the electric field is smaller than that of device R504 and device R640. The carriers in device R505 need longer time to pass through its InGaAs depletion region. Therefore, the trapping efficiency is higher. According to eqn (7), the collector current should be smaller.

\section{CONCI.USION}

In this study, DHBTs with $\operatorname{In}_{0.52} \mathrm{Al}_{x} \mathrm{Ga}_{0.48-x} \mathrm{As}$ collector are investigated. From the Gummel plots of the devices with the $2 \mathrm{DEG}$ effect, the relation between the collector current and the 2DEG density is found to be an exponential function which is mainly resulted from the thermal emission escaping process of $2 \mathrm{DEG}$ charges. It is also found that the conduction band offset is a main factor to control the escaping barrier.

Acknowledgements - This study was partially supported by the National Science Council of the Republic of China under contract No. NSC-82-0404-E002-244 and the Telecommunication laboratory, Ministry of Communication of the Republic of China under contract No. TLNSC-82-5107.

\section{REFERENCES}

1. Q. Wang, Y. Wang, K. F. Longenbach, E. S. Yang and W. I. Wang, Appl. Phys. Lett. 59, 2582 (1991). 
2. Q. Wang, E. S. Yang, Y. K. Chen, D. Sivco and A. Y. Cho, Appl. Phys. Lett. 62, 3129 (1993).

3. C. H. Huang and H. H. Lin, Solid-St. Electron. 36, 1229 (1993).

4. S. C. Lee, J. N. Kau and H. H. Lin, Appl. Phys. Lett. 45, 1114 (1984)

5. C. Z. Chen and S. C. Lee, IEEE Trans. Electron Devices ED-34, 1463 (1987).
6. S. C. Lee and H. H. Lin, J. appl. Phys. 59, 1688 (1986).

7. S. Tiwari, IEEE Electron Device Lett. 9, 142 (1988).

8. C. T. Kirk, IRE Trans. Electron Devices ED-3, 164 (1962).

9. S. Tiwari, Compound Semiconductor Device Physics, p. 234. Academic Press, New York (1992).

10. S. Tiwari, Compound Semiconductor Device Physics, p. 415. Academic Press, New York (1992). 